\title{
Confiance dans le système bancaire et croissance économique
}

\author{
Bruno Amable* \\ Jean-Bernard Chatelain** \\ Olivier De Bandt***
}

Cet article propose un modèle de croissance endogène dans lequel on introduit des intermédiaires financiers en concurrence imparfaite et une rémunération du capital soumise à un choc macroéconomique. Les anticipations rationnelles des ménages sur le risque de faillites bancaires (dont ils supportent en partie les coûts) influencent leurs décisions de dépôts, contribuent à déterminer le taux de croissance, et peuvent être à l'origine d'un piège à pauvreté. La réglementation de l'entrée dans le secteur bancaire peut, dans certains cas, permettre un meilleur arbitrage entre l'efficacité et la stabilité du secteur bancaire, et, en conséquence, augmenter le nombre de dépôts, la croissance et le bien-être.

\section{CONFIDENCE IN THE BANKING SYSTEM AND ECONOMIC GROWTH}

This paper présents a model of endogenous growth with imperfect competition in the banking sector and macroeconomic uncertainty. Households have rational expectations of banks failures, whose costs affect their deposit behaviour and the growth rate and may cause a self-fulfilling poverty trap. The régulation of entry in the banking sector may provide a better tradeoff between stability and efficiency than free entry, and increase the number of deposits, growth and welfare.

Classification JEL : 016

* INRA et CEPREMAP.

** Banque de France, 41-1391 Centre de recherche, F-75049 Paris Cedex 01, France. E-mail. frbdf4pg@ibmmail.com.

*** Banque de France, Centre de recherche.

Les opinions émises dans cet article ne sont pas nécessairement celle de la Banque de France. Nous remercions, sans les impliquer, Bernard Bensaïd et Philippe Madiès pour leurs commentaires. 


\section{INTRODUCTION}

En facilitant les transactions, en collectant l'épargne et en intervenant dans l'allocation du capital vers les secteurs les plus productifs, les intermédiaires financiers jouent un rôle important dans les conditions de la formation de l'équilibre entre épargne et investissement, et, de là, dans la croissance économique ${ }^{1}$. Comme l'illustre le cas de certains pays d'Asie à forte croissance au cours des dernières décennies, une épargne domestique importante peut faciliter le développement économique (World Bank [1993]), compte tenu des insuffisances observées des flux de capitaux internationaux vers les pays moins développés ${ }^{2}$. Le niveau de l'épargne domestique est affecté inter alia par l'importance des déficits publics, le type de système de sécurité sociale, la répartition des revenus, le développement du crédit à la consommation et les taux d'intérêt. Ces derniers peuvent être plafonnés par les pouvoirs publics mais, plus généralement, résultent de la concurrence souvent imparfaite entre intermédiaires financiers, qui se différencient par la localisation, les relations de clientèle et la variété des services offerts. Les frictions issues de l'existence de ces monopoles locaux peuvent cependant être atténuées par diverses interventions publiques, qu'on peut modéliser sous une forme générale d'infrastructures financières ${ }^{3}$ (Amable et Chatelain [1996]). Le développement de ces biens publics plus ou moins spécifiques au secteur financier peut améliorer la collecte de l'épargne, faciliter l'investissement et permettre une croissance plus forte.

Dans cet article, nous mettons l'accent sur un bien public particulièrement important, la confiance des épargnants dans le système bancaire. Ce facteur a certainement joué un rôle aux Etats-Unis, caractérisés par la récurrence des paniques bancaires durant la période 1850-1913 et pendant la crise des années trente où elles ont sans doute contribué à la chute de la croissance économique. Ce phénomène est illustré par la corrélation entre la croissance économique et un indicateur de la défiance envers les intermédiaires financiers tels que le ratio monnaie fiduciaire/dépôts (Friedmann et Schwartz [1965]). Dans nombre de pays peu développés, la confiance des déposants dans le système financier n'est pas acquise, du fait d'une réglementation peu efficace et d'un risque important de défaut des banques (Fry [1995]).

Nous montrons comment les anticipations rationnelles des ménages sur le risque des faillites bancaires (dont ils supportent en partie les coûts, car ils sont en situation d'asymétrie d'information vis-à-vis des intermédiaires financiers), jouent un rôle important dans la collecte de l'épargne et peuvent plonger l'économie dans un piège à pauvreté. La réglementation de l'entrée dans le secteur bancaire peut permettre un meilleur arbitrage entre l'efficacité et la stabilité du

1. Voir Amable et Chatelain [1995] pour une revue des travaux récents sur ce sujet.

2. Toutefois, il existe des causalités réciproques entre l'épargne, l'investissement et la croissance. Carroll et Weil [1994] montrent que la croissance cause au sens de Granger l'épargne plutôt que l'inverse.

3. On peut citer les infrastructures de transport et de télécommunication, le niveau d'éducation, la préservation et la stabilité des droits de propriétés, les infrastructures spécifiques intervenant dans la rapidité et de la fiabilité du système de paiement (chèques, cartes de crédit), et une réglementation efficace évitant les paniques bancaires et instaurant la confiance des déposants. 
secteur bancaire, et, en conséquence, augmenter le nombre de dépôts, l'épargne intermédiée, la croissance et le bien-être. Par exemple, la réglementation de l'entrée dans le secteur bancaire au Canada a maintenu un oligopole entre 1920 et 1980, dont la stabilité est allée de pair avec une efficacité supérieure au système américain où la réglementation s'est efforcée d'éviter la concentration bancaire (Bordo, Rockoff et Redish [1994]) ${ }^{1}$. Dans de nombreux pays, l'État réglemente l'entrée dans le système bancaire ainsi que la rémumération des dépôts, de telle sorte que le nombre d'intermédiaires financiers n'est plus nécessairement déterminé par une condition de libre entrée, mais par le critère choisi par l'organisme réglementant le système bancaire, qui, de manière normative, devrait maximiser le bien-être des ménages ${ }^{2}$.

La seconde section de cet article décrit le comportement des entreprises, des ménages et des intermédiaires financiers. La troisième section présente les différents régimes de croissance possibles. La quatrième section étudie les effets de l'instauration de barrières à l'entrée (restriction du nombre de banques ou réglementation du taux de rémunération des dépôts) et la dernière section conclut l'article.

\section{LE MODÈLE}

\section{Les entreprises}

La technologie est caractérisée par des rendements constants par rapport au capital $\mathrm{k}$ et au travail $\mathrm{N}$, dans une spécification Cobb-Douglas. Le capital est déprécié intégralement d'une période à une autre, hypothèse courante mais non indispensable dans les modèles à générations imbriquées. La population active $\mathrm{N}$ est constante.

$$
y_{t}=a_{t} \cdot k_{t}^{\alpha} \cdot \mathrm{N}_{t}^{1-\alpha}
$$

Le terme de productivité $a$ présente une externalité positive, fonction du capital privé agrégé $\mathrm{K}_{t}$ comme dans Romer [1986] :

$$
a_{t}=\mathrm{A} \cdot \mathrm{K}_{t}^{1-\alpha}
$$

Les entreprises sont en concurrence parfaite sur le marché des biens. Elles font face à un choc macroéconomique sur la production (identique pour toutes les entreprises), réalisé à la période suivante et noté $u_{t+1}$. Il est indépendam-

1. Le système bancaire canadien n'a connu une seule faillite bancaire entre 1920 et 1980, en résistant notamment à la crise financière de 1929.

2. Besanko et Thakor [1992] étudient également l'effet des barrières à l'entrée dans le secteur bancaire dans un modèle d'équilibre partiel qui n'intègre pas les effets sur la croissance économique. D'autres hypothèses diffèrent du présent modèle : ils supposent une concurrence spatiale sur la marché du crédit, la probabilité de défaut bancaire est exogène (ils considèrent deux états de la nature), les anticipations des déposants ne sont pas prises en compte, mais ils supposent la présence d'actionnaires financant le capital des banques et font intervenir le ratio dette/fonds propres des banques. Lorsque les barrières à l'entrée décroissent, le bien-être des actionnaires des banques diminue alors que celui des déposants et des emprunteurs augmente. 
ment et identiquement distribué sur $[\underline{u}, \bar{u}]$ d'une période à une autre, d'espérance $\mathrm{E}_{t}\left[u_{t+1}\right]=1$. Les entreprises choisissent les facteurs de production en maximisant l'espérance de leurs profits :

$\left(\mathrm{N}_{t+1}^{*}, \mathrm{~K}_{t+1}^{*}\right) \in \operatorname{ArgMax} \mathrm{E}_{t}\left[u_{t+1} \cdot a_{t+1} k_{t+1}^{\alpha} \mathrm{N}_{t+1}^{1-\alpha}-w_{t+1} \mathrm{~N}_{t+1}-\tilde{\mathrm{R}}_{t+1} k_{t+1}\right]$

Pour simplifier, nous supposons que le marché du travail est en concurrence parfaite, que les salariés sont prioritaires par rapport aux détenteurs du capital, et que la borne inférieure de l'aléa vérifie $\underline{u} \geqslant 1-\alpha$ : ils recoivent une rémunération certaine, notée $w_{t+1}$. La rémunération aléatoire du capital est $\tilde{\mathbf{R}}_{t+1}$, dont nous supposerons qu'elle est distribuée suivant la fonction de répartition $F$. Les demandes de facteurs sont données par les relations habituelles entre le coût marginal et la productivité marginale. Le dernier membre de l'égalité correspond aux productivités marginales de la production agrégée après la prise en compte de l'externalité (nous éliminons l'indice temporel lorsqu'il n'est pas nécessaire).

$$
\begin{aligned}
& \mathrm{E}_{t}\left[\tilde{\mathrm{R}}_{t+1}\right]=a_{t+1} \cdot \alpha \cdot k_{t+1}^{\alpha-1} \cdot \mathrm{N}_{t+1}^{1-\alpha}=\mathrm{A} \cdot \alpha \cdot \mathrm{N}^{1-\alpha} \\
& w_{t+1}=a_{t+1} \cdot(1-\alpha) \cdot k_{t+1}^{\alpha} \cdot \mathrm{N}_{t+1}^{-\alpha}=\mathrm{A} \cdot(1-\alpha) \cdot \mathrm{K}_{t+1} \cdot \mathrm{N}^{-\alpha}
\end{aligned}
$$

\section{Le comportement des ménages et le marché des dépôts}

Nous considérons un modèle simple à générations imbriquées. La population d'une génération est de taille fixe et vit deux périodes. Elle est répartie sur un continuum de masse normalisée à $\mathrm{N}$, et distribuée uniformément sur un cercle de longueur $L$ (leur densité en un point du cercle est $N / L$ ). Les ménages sont neutres vis-à-vis du risque : leur fonction d'utilité dépend de leur consommation à chaque période sous une forme linéaire. Nous supposons qu'ils n'ont pas un accès direct aux marchés financiers et qu'ils ne peuvent pas utiliser leur épargne pour créer une entreprise et s'autofinancer. À la fin de la première période, chaque ménage recoit un salaire pour l'unité élémentaire de travail qu'il a offert. Il choisit le montant de son épargne $S_{t}$ et son affectation à l'actif sans risque $\left(b_{t}=0\right)$ ou à l'actif risqué $\left(b_{t}=1\right)$, en prenant en compte l'espérance de rendement net des coûts de transaction pour chacun des actifs :

$\left(\mathrm{S}_{t}, b_{t}\right) \in \operatorname{Arg} \max \left(w_{t}-\mathrm{S}_{t}\right)+\frac{1}{1+\rho} \mathrm{E}_{t}\left(b_{t}\left[\left(\tilde{\mathrm{R}}_{t+1}^{\mathrm{IF}}-d l\right)+\left(1-b_{t}\right) v\right] \mathrm{S}_{t}\right)$

$\rho$ est le taux de préférence pour le présent, $v$ est le rendement de l'actif sans risque obtenu par une technologie de stockage, dont le rendement est inférieur à l'espérance du rendement de l'actif productif $\mathrm{E}_{t}\left[\tilde{\mathrm{R}}_{t+1}\right]$. Le rendement aléatoire d'un dépôt chez un intermédiaire financier, qui peut faire défaut, est noté $\tilde{\mathrm{R}}_{t+1}^{\mathrm{IF}}$. Les ménages font face à un coût de «transport » associé au dépôt, proportionnel à la distance $l$ à l'intermédiaire financier, de coût unitaire $d$. Cette hypothèse rend compte de la différenciation horizontale entre les intermédiaires financiers ${ }^{1}$, identique à celle du modèle de Salop [1979]: $n$ intermédiaires

1. On peut en donner différentes interprétations : le coût d'opportunité du temps passé pour se rendre à la banque, la nature des services offerts aux déposants, comme la taille de leur réseaux de distributeurs automatiques, les facilités d'accès au crédit à la consommation, la densité de leurs implantations à l'étranger, la qualité de leurs prestations (accueil, gestion des comptes), etc. 
financiers sont localisés de manière équidistantes sur le cercle. Du fait de l'hypothèse de neutralité vis-à-vis du risque, un ménage épargnera l'intégralité de son revenu $\left(\mathrm{S}_{t}=w_{t}\right)$ si l'espérance du rendement de l'épargne est supérieure à son taux de préférence pour le présent, c'est-à-dire max $\left[v, \mathrm{E}_{t}\left[\tilde{\mathrm{R}}_{t+1}^{\mathrm{IF}}\right]-d \cdot l\right]$ $>1+\rho$, ce que nous supposons vérifié pour tous les ménages. La propension à épargner individuelle ne dépend donc pas du taux d'intérêt. Ce comportement simple de l'épargne individuelle nous permet de développer un comportement original de l'épargne intermédiée.

Nous supposons que les ménages ne peuvent pas vérifier ex post (à la date $t+1$ ) les rendements des crédits octroyés par les banques aux entreprises sur la base de leurs dépôts collectés à la date $t$. Ils connaissent cependant la distribution de probabilité du rendement $e x$-ante. Une banque $i$ offre un taux d'intérêt sur les dépôts $r_{i}$ qui prend la forme d'un contrat de dette, où l'intermédiaire financier subit un coût de faillite non pécuniaire, suivant l'hypothèse du modèle de Diamond [1984]. Ces coûts de faillite correspondent au temps passé par les intermédiaires dans les procédures de faillite, les coûts de recherche d'un nouveau gestionnaire de la banque ${ }^{1}$. Si les revenus de la banque ne remboursent pas la valeur de la dette, elle déclare la faillite. D'autre part, nous supposons que les déposants ne reçoivent pas la valeur restante de l'intermédiaire financier en cas de défaut. Le montant de ces fonds est intégralement absorbé par des coûts de faillite à la charge du déposant.

\section{Équilibre avec libre entrée des banques}

La séquence de l'équilibre est la suivante. Les épargnants disposent tous d'une même croyance a priori sur la probabilité de succès $p^{2}$. Comme nous nous intéressons à la confiance globale dans le système bancaire, nous la supposons identique pour toutes les banques. Ceci traduit d'une manière condensée la corrélation entre les faillites bancaires en chaîne lors d'un mouvement de panique des déposants. Du fait des coûts de faillite bancaire, les ménages anticipent de ne recevoir aucun revenu avec une probabilité $1-p$, donc $E_{t}\left[\tilde{\mathrm{R}}_{t+1}^{\mathrm{IF}}\right]=p \cdot r_{i}{ }^{3}$. Les banques, connaissant cette croyance, déterminent le taux sur les dépôts. Compte tenu du niveau de ces taux, les ménages déposent leur épargne dans la banque $i$ si le rendement attendu du dépôt net du coût de transport est supérieur au rendement de l'actif sans risque $\left(p \cdot r_{i, t}-d \cdot l \geqslant v\right)$. Si un montant suffisant d'épargne a pu être collecté, les banques investissent les fonds collectés dans des entreprises et paient un coût fixe de collecte. Elles obtiennent à la période suivante le rendement de leur crédits aux entreprises, et, sauf en cas de faillite, effectuent les remboursements aux déposants. Un premier équilibre, pour un nombre de banques fixé, est obtenu en supposant que les anticipations

1. Formellement, la banque subit une pénalité non pécuniaire endogène (i.e. déterminée par le contrat optimal), qui la laisse indifférente par rapport au cas où elle aurait à payer le taux $r_{i}$ promis aux déposants. Si elle décide de rembourser à un taux inférieur $\mathrm{z}$, elle subira une pénalité $\phi(z)=\max \left(r_{i}-z, 0\right)$ (Diamond [1984]).

2. Un modèle alternatif pourrait modéliser l'hétérogénéité des croyances des déposants par le paramètre de différenciation horizontale.

3. Matutes et Vives [1996] envisagent aussi le cas où les banques peuvent influencer les anticipations des déposants par le choix de leur taux sur les dépôts. 
des ménages sont rationnelles et autoréalisatrices. Enfin, on détermine l'équilibre de libre entrée des banques (profits nuls) ou la décision de réglementation de l'entrée.

Pour une anticipation de succès $p \in[0,1]$, les banques maximisent l'espérance de leurs profits, sans qu'ils soient conditionnels à l'absence de faillite, du fait de la présence des pénalités non pécuniaires. La banque doit attirer un montant minimal de dépôts pour pouvoir investir, compte tenu d'un coût fixe de collecte noté $\mathrm{C}_{t}$. En raison de leur neutralité vis-à-vis du risque, les banques investissent tous leurs fonds dans les actifs productifs et risqués, sans asymétrie d'information, et rien dans l'actif non risqué moins rentable. Elles n'ont pas de fonds propres. Nous supposons que la concurrence sur les marchés des dépôts et du crédit est imparfaite et déterminée par un équilibre de Cournot-Nash à $n$ joueurs. Côté dépôts, il y a différenciation horizontale et l'élasticité de la demande est nulle et, côté crédit, il n'y a pas de différenciation horizontale mais l'élasticité de la demande est supérieure à l'unité, en valeur absolue. La banque choisit ex ante le montant de crédit aux entreprises et le montant de dépôts offerts aux ménages ${ }^{1}$ :

$$
\begin{aligned}
\left(\mathrm{D}_{i, t}, k_{i, t+1}\right) & \in \operatorname{Argmax} \mathrm{E}_{t}\left[\Pi_{i}\right]=\mathrm{E}_{t}\left[\tilde{\mathrm{R}}_{i, t+1}\right] \cdot k_{i, t+1}-r_{i, t} \cdot \mathrm{D}_{i, t}-\mathrm{C}_{t} \\
\text { s.c. } k_{i, t+1} & =\mathrm{D}_{i, t} \\
R_{i, t+1} & =\mathrm{E}_{t}\left[\tilde{\mathrm{R}}_{i, t+1}\right]=\alpha \cdot a \cdot k_{t+1}^{\alpha-1} \cdot \mathrm{N}^{1-\alpha} \\
\mathrm{D}_{i, t} & =2 \cdot \frac{\mathrm{N}}{\mathrm{L}} \cdot w_{t} \cdot p \cdot \frac{r_{i, t}-(\mathrm{v} / p)}{d}
\end{aligned}
$$

Ce choix se fait sous les contraintes d'équilibre comptable de la banque, de connaissance de la courbe de demande de crédit agrégée et de la demande de dépôts $\mathrm{D}_{i, t}$ spécifique à la banque $i$. En effet, l'emprunteur marginal, indifférent entre déposer son épargne à la banque $i$ et investir dans l'actif sans risque, est localisé à une distance $l(p, r)=\frac{p \cdot r_{i}-v}{d}$ de la banque. Les dépôts collectés par la banque $i$ sont donc $2 \cdot l$, multipliés par la densité des déposants par unité de distance $\mathrm{N} / \mathrm{L}$ et par leur épargne individuelle $w_{t}$. Nous avons donc supposé que le nombre de banques ( $n$ ) est tel que la distance entre deux banques est toujours supérieure à $2 \cdot l$ si bien que les marchés des dépôts ne se recoupent pas (monopoles locaux «purs ») : $\frac{L}{n} \geqslant 2 \cdot l$, ce qui devra être vérifié par la suite.

À l'équilibre symétrique, le taux d'équilibre sur les dépôts est le même pour toutes les banques :

$$
r=\frac{1}{2}\left\{\mathrm{E}_{t}\left[\tilde{\mathrm{R}}_{t+1}\right]\left[1+\frac{\alpha-1}{n}\right]+\frac{v}{p}\right\} \Rightarrow n(p, r)=\frac{(1-\alpha) \mathrm{E}_{t}\left[\tilde{\mathrm{R}}_{t+1}\right]}{\mathrm{E}_{t}\left[\tilde{\mathrm{R}}_{t+1}\right]-2 \cdot r+\frac{v}{p}}
$$

\footnotetext{
1. Lorsqu'il n'y a pas de défaut ex post, nous supposons par commodité que le surplus $\left(\tilde{R}_{i, t+1}-E_{t}\left[\tilde{R}_{i, t+1}\right]\right) k_{i, t+1}$ est consommé par l'intermédiaire financier.
} 
C'est une moyenne du taux sur le crédit (diminué d'un taux de marge sur le crédit, lui-même fonction de l'élasticité de la demande de crédit et du nombre de concurrents, du fait de la concurrence à la Cournot), et du rendement de l'actif alternatif corrigé par la probabilité de succès du système bancaire. Une augmentation de la confiance anticipée des déposants dans le système financier $p$ augmente la marge de concurrence imparfaite.

Cet équilibre impose que les anticipations des déposants soient rationnelles. La probabilité de succès satisfait la condition suivante :

$$
\left.p=1-\operatorname{Pr} \tilde{\mathrm{R}}_{t+1} \cdot k_{t+1}-r_{t+1} \cdot \mathrm{D}_{t}-\mathrm{C}_{t} \leqslant 0\right)=1-\mathrm{F}\left(r_{i}+c\right)
$$

Nous supposons ici que le coût fixe d'intermédiation est proportionnel à la taille des dépôts dans chaque banque : $\mathrm{C}_{t}=c \cdot \mathrm{D}_{i, t}^{*}$. Ce genre d'hypothèse est nécessaire dans un modèle de croissance, afin que les coûts fixes ne deviennent pas négligeables par rapport à la production. La probabilité de faillite augmente avec la taille du coût fixe.

Le système des deux dernières équations donne les valeurs de l'équilibre ( $p^{*}$, $\left.r^{*}\right)$ à nombre de banques fixé. S'il y a libre entrée dans le secteur bancaire, le nombre de banques, $n$, va s'accroître à long terme jusqu'au point où les profits sont nuls. En ignorant la contrainte de nombre entier, en normalisant la population à l'unité $(\mathrm{N}=1$, donc la densité des ménages en un point du cercle devient $1 / \mathrm{L})$, et en prenant en compte l'externalité dans le secteur productif $\left(\mathrm{E}_{t}\left[\tilde{\mathrm{R}}_{t+1}\right]=\mathrm{A} \cdot \alpha\right)$ la condition d'espérance de profit nul pour les banques ajoute une troisième équation qui donne les valeurs $\left(p^{* *}, r^{* *}, n^{* *}\right)$ caractérisant l'équilibre avec libre entrée des intermédiaires financiers :

$$
\begin{aligned}
r^{* *} & =\mathrm{A} \cdot \alpha-c \\
p^{* *} & =1-\mathrm{F}(\mathrm{A} \cdot \alpha)=1-\mathrm{F}\left[\mathrm{E}_{t}\left[\tilde{\mathrm{R}}_{t+1}\right]\right] \\
n\left(p^{* *}, r^{* *}\right) & =\frac{\mathrm{A} \cdot \alpha \cdot(1-\alpha)}{2 \cdot c+\frac{v}{1-\mathrm{F}(\mathrm{A} \cdot \alpha)}-\mathrm{A} \cdot \alpha}
\end{aligned}
$$

L'augmentation du coût fixe d'intermédiation $c$ ou du rendement de l'actif alternatif $v$ réduit le nombre de banques et accroît la concurrence imparfaite dans le secteur bancaire. La condition d'espérance de profit nul impose une probabilité de faillite élevée, égale à la probabilité que le rendement du crédit soit inférieur à son espérance ( $1 / 2$ lorsque la variable aléatoire est symétrique).

\section{TROIS RÉGIMES DE CROISSANCE}

Nous rappelons que les entreprises obtiennent des fonds uniquement par l'intermédiation financière. Nous avons supposé que le capital se déprécie intégralement d'une période à une autre. En conséquence, le stock de capital est égal à l'investissement qui est lui-même déterminé par l'épargne de la jeune génération collectée par les banques. Trois régimes sont possibles. 


\section{Piège à pauvreté}

Il apparaît de manière « autoréalisatrice » lorsque les ménages anticipent de façon certaine la faillite du système bancaire $(p=0)$ quels que soient l'espérance de la productivité des entreprises et les coûts d'intermédiation. Les banques n'ont alors aucun déposant quel que soit le taux d'intérêt qu'elles offrent sur les dépôts. Du fait de la défiance des déposants, il n'y a donc pas d'intermédiation financière, alors qu'elle est le seul moyen de financer la croissance en établissant la liaison entre les épargnants et les emprunteurs.

Si les anticipations des ménages sont telles que $p \in] 0,1]$, aucun épargnant ne dépose à la banque lorsque le rendement du capital $\mathrm{A} \cdot \alpha$ est insuffisant par rapport au niveau des coûts d'intermédiation et du rendement alternatif de la technologie de stockage, accru de l'anticipation autoréalisatrice de faillite bancaire (condition technique : $l^{* *}=0 \Rightarrow A \cdot \alpha<c+\left(v /\left(p^{* *}\right)\right)$. L'épargne collectée par les banques est alors nulle, et le secteur productif n'est pas financé.

\section{Monopoles locaux}

Ils existent pour les conditions techniques suivantes $0<l^{* *}\left(p^{* *}, r^{* *}\right)<\mathrm{L} / 2 \mathrm{n} * *$ et $n^{* *}>0$. Cela implique que le rendement du capital $\mathrm{A} \cdot \alpha$ est borné : $c+\left(v / p^{* *}\right)<\mathrm{A} \cdot \alpha<\min \left(2 \cdot c+\left(v / p^{* *}\right), \mathrm{H}\left(c, v / p^{* *}\right)\right)$, où $\mathrm{H}$ est la solution la plus élevée de l'équation du second degré en $\mathrm{A} \cdot \alpha$ associée à $21^{* *} n^{* *}=\mathrm{L}$. L'existence dans le long terme d'un équilibre où le marché des dépôts n'est pas intégralement couvert apparaît en raison de la concurrence imparfaite sur le marché du crédit. Sinon, une banque pourrait toujours entrer sur la zone non servie par les autres banques, jusqu'à ce que toutes les banques soient en concurrence directe pour le déposant marginal. Le facteur de croissance est donné par :

$$
\begin{aligned}
\mathrm{G}_{t} & =\frac{\mathrm{K}_{t+1}}{\mathrm{~K}_{t}}=n^{*} \cdot\left(2 \cdot l^{*}\right) \cdot \frac{w_{t}}{\mathrm{~K}_{t}} \cdot \frac{1}{\mathrm{~L}}=\frac{n^{*} \cdot\left(2 \cdot l^{*}\right)}{\mathrm{L}} \cdot \mathrm{A} \cdot(1-\alpha) \\
& =\frac{2 \mathrm{~A}^{2}(1-\alpha)^{2} \alpha[1-\mathrm{F}(\mathrm{A} \cdot \alpha)]\left[\mathrm{A} \cdot \alpha-\left(c+\frac{v}{[1-\mathrm{F}(\mathrm{A} \cdot \alpha)]}\right)\right]}{d \mathrm{~L}\left[2 c+\frac{v}{1-\mathrm{F}(\mathrm{A} \cdot \alpha)}-\mathrm{A} \alpha\right]} \\
& =\mathrm{G}\left(\begin{array}{l}
\mathrm{A}, d, c, v, p^{* *}, \alpha, \mathrm{L} \\
+--++?
\end{array}\right)
\end{aligned}
$$

L'intermédiation financière intervient dans la détermination du taux de croissance par le nombre de banque $n^{* *}$ et la part de marché de chaque banque mesurée par la distance $2 \cdot l^{* *}$, qui accroissent le nombre de déposants. Le taux de croissance est constant. Comme dans le modèle de croissance endogène dit A.K, il dépend positivement de la productivité du capital. La concurrence imparfaite dans le secteur bancaire ajoute trois nouvelles variables explicatives aux effets négatifs sur la croissance : la densité de population par unité de longueur $(1 / \mathrm{L})$, le coût d'intermédiation des banques $c$ et le rendement de l'actif alternatif, augmenté du risque de faillite des banques $v / p^{* *}$. 


\section{Collecte complète de l'épargne}

La condition est la suivante : $l^{* *}=\mathrm{L} / 2 n^{* *}$, ce qui implique que le rendement du capital $\mathrm{A} \cdot \alpha$ est supérieur à un certain niveau $\mathrm{A} \cdot \alpha>\mathrm{H}\left(c, v / p^{* *}\right)$. Comme l'intégralité des salaires est collectée, l'égalité épargne-investissement donne le facteur de croissance (maximal) : $\mathrm{G}_{t}=\frac{w_{t}}{\mathrm{~K}_{t}}=\mathrm{A} \cdot(1-\alpha)$.

\section{RÉGLEMENTATION DE LA CONCURRENCE}

Les déposants subissent un coût en cas de faillite bancaire. Dans le cas des équilibres qui résultent d'anticipations des ménages permettant un fonctionnement des banques $(p \in] 0,1])$ une politique réglementaire limitant l'entrée et diminuant la probabilité de faillite des banques peut réduire l'espérance des coûts de faillite, par rapport à un éventuel « excès » de concurrence provenant de la condition de libre entrée. Une mesure du bien-être de la population d'agents hétérogènes (quant au rendement de leur épargne, fonction de leur localisation) est donnée par la somme $\mathrm{U}^{a}$ des utilités d'une génération :

$$
\mathrm{U}^{a}=\frac{1}{\mathrm{~L}} \frac{w_{t} \cdot 2 \cdot n(r, p)}{(1+\rho)}\left\{\int_{0}^{l}[p \cdot r-d \cdot i] d i+\int_{l}^{L /(2 \cdot n(r, p))}[\mathrm{v}] d i\right\}
$$

Le choix du nombre de banques maximisant le bien-être, sous les deux contraintes définissant l'équilibre à nombre de banques donné, revient, après avoir substitué la contrainte définissant $n(r, p)$, à choisir une réglementation du taux des dépôts maximisant le bien-être :

$$
\begin{gathered}
\max _{r} \mathrm{U}^{a}(r, p)=\max _{r}\left[n(r, p) \cdot(p \cdot r-\mathrm{v})^{2}+\mathrm{v} \cdot d\right] \cdot n(r, p) \cdot(p \cdot r-\mathrm{v}) \\
\text { s.c. } p=1-\mathrm{F}(r+c) \\
\frac{v}{1-F(r+c)} \leqslant \mathrm{r} \leqslant \mathrm{A} \cdot \alpha-c \\
\frac{\mathrm{A} \cdot \alpha \cdot(1-\alpha)}{2 \cdot c-\mathrm{A} \cdot \alpha+\frac{v}{1-\mathrm{F}(\mathrm{A} \cdot \alpha)}} \leqslant \frac{d \cdot \mathrm{L}}{2 \cdot\{[1-\mathrm{F}(\mathrm{A} \cdot \alpha)] \cdot(\mathrm{A} \alpha-c)-\mathrm{v}\}}
\end{gathered}
$$

L'avant-dernière condition porte sur le taux de rémunération des dépôts. S'il est trop bas par rapport à l'actif sans risque $(l(p, r)=0)$ aucune banque ne peut fonctionner. Sa borne supérieure correspond au cas de la libre entrée, qui donne le nombre de banques maximal. La dernière condition vérifie que le coût fixe est suffisamment élevé pour que le calcul du bien-être établi dans le cas des monopoles locaux puisse s'appliquer. Le nombre de banques est alors insuffisant, même lorsqu'il y a libre entrée, pour couvrir l'intégralité de la circonférence du cercle. $\mathrm{U}^{a}(r, p)$ est une fonction croissante en ses deux arguments $\left(\mathrm{U}_{r}^{a}>0, \mathrm{U}_{p}^{a}>0\right)$. La condition du premier ordre peut s'écrire sous la forme :

$$
\mathrm{U}_{r}^{a}=\mathrm{U}_{p}^{a} \cdot f(r+c)
$$


En termes de bien-être pour les épargnants, une baisse de la rémunération des dépôts (de l'« efficacité ») peut être compensée par une diminution des coûts de faillites (un gain de sécurité et de stabilité du secteur bancaire). On trouve aussi cet effet pour une collecte complète de l'épargne (Amable, Chatelain et De Bandt [1996]). Pour qu'une telle condition soit satisfaite, il faut que l'effet marginal d'une baisse du taux d'intérêt sur la probabilité de succès soit suffisamment élevé, c'est-à-dire que la distribution du risque soit suffisamment concentrée au point $r+c$. Un tel résultat dépend des hypothèses sur la distribution du risque retenue. Dans de nombreux cas, l'accroissement de l'efficacité domine celui de la stabilité $\left(\mathrm{U}_{r}^{a}>\mathrm{U}_{p}^{a} \cdot f(r+c)\right)$ pour les taux d'intérêt admissibles, et l'équilibre de libre entrée représente la meilleure solution. Nous donnons un exemple où la maximisation du bien-être peut toutefois conduire à limiter l'entrée de nouvelles banques, avec $\mathrm{L}=1, \mathrm{~A}=4.2, \alpha=0.36, c=0.26, v=1, d=0.2$. La fonction de répartition correspond à la variable aléatoire $\tilde{R}=\mathrm{E}_{t}\left[\tilde{\mathrm{R}}_{t+1}\right]-0.5+\mathrm{X}$, où $\mathrm{X}$ suit une loi béta $\beta(2,4)$ définie sur l'intervalle $[0,1]$. Dans ce cas, le taux de rémunération des dépôts avec libre entrée est approximativement de 1.25 (correspondant à $n \simeq 4$ banques), et le taux de rémumération des dépôts maximisant le bien-être est approximativement de 1.179 (correspondant à $n \simeq 3.5$ banques). Le taux de croissance dans le cas de la réglementation de la concurrence bancaire est nettement au-dessus du taux de croissance avec libre entrée $(4,7>1,9)$, mais très légèrement en dessous du taux de croissance maximal possible, obtenu pour un taux de rémunération des dépôts à peine plus élevé.

\section{CONCLUSION}

Cet article montre comment les anticipations autoréalisatrices des ménages sur le risque de faillite du système bancaire peut plonger l'économie dans un piège à pauvreté. La présence de coûts de faillites bancaires supportés par les déposants justifie, dans certains cas, une réglementation de l'entrée dans le secteur bancaire : le gain en stabilité du système financier compense alors la baisse de rémunération des dépôts. La réglementation peut augmenter le nombre de déposants, le montant de l'épargne intermédiée, la croissance et le bien-être. L'effet de la concurrence imparfaite sur la croissance joue ici sur le nombre de déposants et non par un effet prix direct sur la rémunération de l'épargne d'un ménage représentatif. Les variables explicatives du taux d'épargne et du taux de croissance sont le nombre de banques par habitant, les coûts fixes de gestion et de contrôle à l'origine des rendements croissants de l'activité d'intermédiation, le coût d'opportunité des dépôts provenant de la rémunération de l'actif alternatif, le paramètre donnant l'intensité de la différenciation horizontale sur le marché des dépôts, et enfin la probabilité de faillite des intermédiaires financiers perçue par les épargnants, qui rend compte de leur confiance.

Restreindre la concurrence ou réglementer les taux sur les dépôts ne sont pas les seules façons de diminuer les faillites bancaires et les coûts qui leur sont associés. Une assurance sur les dépôts qui garantit que les banques ne seront jamais déclarées en faillite constitue une alternative (Amable, Chatelain et De Bandt [1996]). La prise en compte d'asymétrie d'information entre les banques et l'organisme de supervision implique que le maintien d'un oligopole bancaire 
doit aussi s'accompagner d'autres interventions réglementaires (prêteur en dernier ressort, ratios de solvabilité, contrôles sur place) pour maintenir dans le long terme la confiance des épargnants dans le système bancaire.

\section{RÉFÉRENCES BIBLIOGRAPHIQUES}

Amable B., Chatelain J.B. [1995], « Efficacité des systèmes financiers et développement économique », Économie internationale, 61, p. 99-130.

AmaBle B., Chatelain J.B. [1996], « Financial Infrastructures and Growth », mimeo.

Amable B., Chatelain J.B. et De Bandt O. [1996], « Confidence in the Banking Sector and Economic Growth », mimeo.

Bordo M.D., RockofF H. et Redish A. [1994], «The U.S. Banking System From a Northern Exposure : Stability versus Efficiency », The Journal of Economic History, 54 (2), p. 325-341.

BESANKO D., THAKOR A.V. [1992], « Banking Deregulation : Allocational Consequences of Relaxing Entry Barriers », Journal of Banking and Finance, 16, p. 909-932.

CARroll C., WeIL D. [1994], «Saving and Growth : a Reinterpretation », CarnegieRochester Series on Public Policy, 40, p. 133-192.

DIAMOND D. [1984], « Financial Intermediation and Delegated Monitoring », Review of Economic Studies, p. 939-414.

Friedman M., SChWARTZ A. [1965], A Monetary History of the United States, 18671960, Princeton (N.J.), Princeton University Press.

FRY M.J. [1995], Money, Interest and Banking in Economic Development, New York, The John Hopkins University Press.

MATUTES C., VIVES X. [1996], «Competition for Deposits, Fragility and Insurance », Journal of Financial Intermediation, 5, p. 184-216.

ROMER P. [1986], «Increasing Returns and Long Run Growth », Journal of Political Economy, 94 (5), p. 1002-1037.

SALOP S. [1979], «Monopolistic Competition with Outside Goods », Bell Journal of Economics, 10 (1), p. 141-156.

World Bank [1993], The East Asian Miracle. Economic Growth and Public Policy, Oxford, Oxford University Press. 